Meta

Journal des tradlucteurs

Translators' Journal

\title{
Évolution du français au Québec au cours des vingt dernières années
}

\section{Jean Darbelnet}

Volume 20, numéro 1, mars 1975

Vingt ans de traduction. Bilan et prospectives

URI : https://id.erudit.org/iderudit/002473ar

DOI : https://doi.org/10.7202/002473ar

Aller au sommaire du numéro

Éditeur(s)

Les Presses de l'Université de Montréal

ISSN

0026-0452 (imprimé)

1492-1421 (numérique)

Découvrir la revue

Citer cet article

Darbelnet, J. (1975). Évolution du français au Québec au cours des vingt dernières années. Meta, 20(1), 28-35. https://doi.org/10.7202/002473ar d'utilisation que vous pouvez consulter en ligne. 


\section{Évolution du français au Québec au cours des vingt dernières années}

Venant d'assister à un colloque organisé par la Société des écrivains canadiens sur la qualité du français parlé et écrit au Québec, un ancien étudiant de l'Université Laval, aujourd'hui traducteur de profession, déplorait récemment le pessimisme des membres du panel en opposant à leurs dires des exemples précis d'alignement sur le français international. "Chéquer les brakes », « licence » et « retournable », faisait-il observer, sont nettement en recul devant vérifier les freins, permis de conduire et consigné.

Cette mise au point rassurante s'inscrit naturellement dans le bilan qu'on est tenté de dresser du français au Québec par rapport à ce qu'il était vers 1950, et, dans les limites de cet article, lui fournit un bon point de départ.

Il y a vingt ans, le français des Québécois n'était sans doute pas tout à fait le même qu'en 1934, mais on a tout lieu de supposer que l'évolution a été beaucoup plus rapide au cours des vingt dernières années que pendant les deux décennies précédentes, lesquelles, rétrospectivement, se fondent dans une longue période de quasi-immobilité et pour la langue et pour la société qui s'y reflétait.

Si le français s'est considérablement modifié depuis vingt ans, c'est de toute évidence parce qu'il a suivi - et d'ailleurs ne pouvait pas ne pas suivre l'évolution socio-économique du Québec au cours de la même période.

Avant d'entrer dans le détail de cette évolution, il n'est pas inutile de rappeler les grands traits du parler québécois en face du français qu'il est commode d'appeler international parce qu'il est commun aux États francophones.

D'abord le franco-québécois, qui n'est pas le joual, a gardé un certain nombre de mots et de tournures qui sont tombés en désuétude en France ou ont changé de sens : jaser pour bavarder, assemblée pour réunion, épargner pour économiser, noirceur pour obscurité, hausser pour augmenter, soixante et huit avec et et vis-à-vis sans $d e$, pour ne donner que quelques exemples.

Mais c'est surtout l'anglicisation qui a été pendant longtemps le trait marquant du français en Amérique du Nord. À première vue, cette caractéristique paraît moins prononcée aujourd'hui du fait que le français européen s'anglicise depuis la guerre. Mais en y regardant de plus près, on constate que le phénomène de l'anglicisation ne prend pas la même forme à Montréal et à Paris. La différence 
essentielle est que le franco-québécois évite d'employer des mots anglais, alors que l'hexagonal les accueille volontiers. On peut parier d'avance qu'en Europe streaking (s'il doit durer) sera préféré à nuvitisme. Cela ne veut pas dire qu'il n'y a pas d'emprunts directs à l'anglais dans l'usage québécois : «scréper », la «track», le «safe », la «shop », «steamer » représentent une catégorie qui est assez étoffée, mais qui se situe à un certain niveau socio-économique. En fait, l'anglicisation du français québécois est surtout d'ordre sémantique et syntagmatique. D'une part, «la poursuite » pour l'accusation, «l'engin » pour la locomotive, «la charrue » pour le chasse-neige, " partir » pour mettre en route, et d'autre part 《talon étagé », "revue de modes», «frein d'urgence », «magasin à rayons » illustrent ces deux classes d'anglicismes en ne donnant qu'une faible idée de leur importance.

Le français québécois se caractérise également par ses contre-anglicismes qui se sont implantés dans l'usage pour barrer la route à des emprunts anglais, tels que noliser, roulotte, traversier, fin de semaine.

Enfin, c'est aussi un trait du franco-québécois que la déformation du sens et de l'emploi de certains emprunts : «correct» (pour d'accord), «frapper » (pour rencontrer), «pas capable 》 (pour ce n'est pas possible).

Dans l'ensemble ces caractéristiques subsistent; les survivances sont à peu près les mêmes, mais certaines catégories d'anglicismes ont, comme nous le verrons, subi des pertes. C'est l'isolement dans lequel a longtemps vécu le Québec, conjugué avec la proximité de l'anglais, qui explique l'anglicisation : pour faire face à de nouveaux besoins d'expression, il était plus facile de franciser tant bien que mal de l'anglais que de chercher à savoir ce qui se disait en. France en pareil cas. Mais cette situation a changé lorsque le Québec est entré en symbiose avec les autres pays de la francophonie. Depuis que les relations avec la France et l'Afrique d'expression française se sont resserrées, l'écart tend à diminuer entre l'usage québécois et le français international et on constate le recul de certaines façons de parler qui apparaissent aujourd'hui comme marginales. Il y a ainsi une refrancisation, et l'histoire du français au Québec depuis vingt ans est en grande partie celle de cette refrancisation. Cela se voit, pour commencer, à certaines inscriptions bilingues. Un autocar qui fait visiter la ville de Québec aux touristes porte l'enseigne suivante : Tour de ville - Sightseeing - Location - Charter. Si cette entreprise existait déjà il y a vingt ans, on peut douter que la raison sociale ait été alors rédigée avec autant de discernement. Il est à remarquer que dans les pages jaunes de l'annuaire du téléphone de Québec on trouve location d'autobus et non «charter». On note aussi, comme donnée négative, que l'usage québécois ne reconnaît pas la distinction que les Français font entre un autobus, un autocar, et un car ; toutefois une firme emploie le mot autocar : «Les Autocars Fournier».

Dans le domaine de la consommation courante, on ne saura jamais si « plumefontaine », très répandu avant 1950, aurait fini par céder la place à stylo, l'emploi généralisé du stylo-bille ayant réglé la question autrement. On trouve maintenant, dans les annonces, complet et pardessus à côté de «habit »et "paletot», modernisation plutôt que refrancisation, puisque les deux termes canadiens ici visés se sont longtemps employés en France. Et il est exact, comme le signalait le traduc- 
teur cité ci-dessus, que les bouteilles de coca cola de la marque COKE portent la mention BOUTEILLE CONSIGNÉE, beaucoup plus brève, en l'occurrence, que son équivalent anglais RETURN FOR BOTTLE REFUND. Il y a là un effort d'autant plus méritoire qu'on a fait appel à la notion de consignation dont le rapport avec la reprise des bouteilles n'est pas évident à première vue.

Les assurances commencent à parler de sinistre, plutôt que de « réclamation », et une compagnie parle même de «boni pour non-sinistre», ce qui peut apparaître comme un excès de zèle. Dans un secteur voisin, celui des assurances sociales (qui s'appelle encore le «bien-être »), le terme avantages sociaux semble l'emporter sur «bénéfices marginaux». C'est ce qui ressort des offres d'emploi que la S.T.Q. diffuse parmi ses membres. Toutefois une offre récente s'arrête à mi-chemin en mentionnant des "bénéfices sociaux ». L'un des obstacles à la refrancisation est la commodité des annonces bilingues prenant appui sur un mot commun aux deux langues : ICE CUBES DE GLACE. Particulièrement édifiant dans ce contexte est le dépliant bilingue d'une carte de crédit titrant, au-dessous de la raison sociale, DEMANDE D'ADHÉSION/APPLICATION. On lui sait gré d'avoir résisté à la tentation d'économiser de la place.

Il y a encore des traducteurs du Québec qui se cramponnent à la désignation «Bureau des gouverneurs », peut-être pour ménager la susceptibilité des membres du «bureau» qui trouvent flatteur d'être appelés «gouverneurs ». Cependant, aux pages financières des journaux, on rencontre assez fréquemment conseìl d'administration, et directeur commence à remplacer "gérant 》 dans la banque. Lorsque l'auteur de ces lignes est revenu au Québec en 1962, le substantif contentieux n'était pas encore entré dans l'usage administratif, peut-être à cause de son paronyme anglais contentious, et on continuait à dire " département légal ». Aujourd'hui on trouve tout naturel, dans nombre d'entreprises, de parler du contentieux, et «département légal 》 devient aussi suranné que «aviseur légal en face de conseiller juridique. "Centre d'achats 》 tient bon, mais centre commercial fait de temps en temps son apparition dans les textes imprimés, et on voit assez souvent SOLDES pour vente à prix réduit. L'édition québécoise n'a pas complètement abandonné « royautés » au profit de droits d'auteurs, et une vieille maison d'édition emploie les deux dans sa correspondance avec ses auteurs. Les motels ont généralement un «OFFICE», le mot ayant l'avantage d'appartenir aux deux langues, sans toutefois avoir le même sens, mais on remarque de temps en temps RECEPTION, qui est le terme exact. $\grave{A}$ ce propos il convient de signaler une initiative ingénieuse pour indiquer si le motel a encore de la place ou est complet. Elle consiste à opposer BIENVENUE à COMPLET sur l'enseigne. Compte tenu du fait que cet emploi de bienvenue n'est pas grammaticalement correct, on doit reconnaître qu'il part d'une louable intention et témoigne d'un souci de refrancisation auquel il convient de rendre justice. Dans les bureaux, «chambre » recule devant porte et local, ce qui représente une amélioration sensible. Heures d'ouverture commence à remplacer « heures d'affaires».

Dans le secteur scolaire, le franco-québécois s'est donné un mot nouveau, cégep, formé à partir d'un sigle, et doté d'un dérivé, cégépien, parallèle à lycéen et à collégien. Il semble qu'au niveau universitaire, on relève moins souvent «octroi », remplacé par subsides ou subvention; c'est ce dernier terme qu'il conviendrait de 
privilégier. Dans au moins deux grandes universités du Québec «bâtisse » a fait place à bâtiment dans TERRAINS ET BÂTIMENTS. Il serait possible de justifier bâtisse au nom d'un ancien usage, mais la langue administrative préfère les mots neutres; or le vocable bâtisse s'entoure d'une certaine coloration qui en fait l'antonyme d'édifice ; bâtiment, se situant entre les deux, est de ce fait préférable. Dans certaines universités les bâtiments s'appellent pavillons quand ils portent un nom destiné à les identifier. Certains se demandent si ces bâtiments ne sont pas trop vastes pour s'appeler ainsi, un pavillon étant souvent de dimensions réduites, par exemple quand il s'agit d'une maison unifamiliale en banlieue. Mais certains corps de bâtiment s'appellent pavillons, et ce mot désigne aussi les unités de construction d'un hôpital moderne, qui sont loin d'être petites.

Traditionnellement, les universités françaises ne font pas de distinction administrative entre les étudiants de licence et ceux de maîtrise ou de doctorat. Il s'ensuit que le français international ne possède pas de terme pour rendre "gradué » comme adjectif ou comme nom. Certaines universités du Québec et de l'Ontario ont remplacé "École des gradués » par «Faculté des études supérieures》, mais Laval. a toujours une "École des gradués». Cependant, l'introduction du mot cycle dans la terminologie universitaire limite dans une certaine mesure l'emploi de "gradué », auquel beaucoup de professeurs se résignent difficilement. On parle donc de premier, second et troisième cycle, des études de premier cycle, encore que «prégradué » se maintienne à cause du nom d'une association. Pour "prérequis », autre anglicisme, on a proposé, non sans quelque succès, préalable, qui, comme substantif a bien le sens de condition prédéterminante.

L'automobile offre plusieurs exemples de refrancisation, dont celui de « vérifier les freins 》, vu plus haut. "Bumper», "cap de roue», «windshield», « tire », « sparks » s'entendent moins souvent qu'il y a vingt ans, dans un domaine où, cependant, l'emploi de catalogues en langue anglaise a été un puissant et durable facteur d'anglicisation. Beaucoup de stations-service affichent encore «gaz » pour essence, mais ce dernier terme est généralement compris et il arrive qu'il figure dans les informations de presse. Parallèlement, on constate que faire le plein fait des progrès dans l'usage québécois. L'expression « école de conduite », caractéristique de cet usage, est correcte et claire, mais cela ne l'empêche pas d'être concurrencée par AUTO-ÉCOLE, qui figure sur certaines des voitures consacrées à ce genre d'apprentissage. Et récemment un article de journal examinait l'éventualité d'un alcootest obligatoire pour tous les automobilistes. Enfin il semble bien que "char » pour voiture ou auto soit en régression. Mais l'expression d'occasion reste rare. Il y a ainsi des mots qui pénètrent difficilement dans le parler des Québécois, comme, par exemple, luge, commune et assesseur.

Le téléphone offre aussi des cas du même genre. En 1963; la compagnie Bell (comme elle s'appelait alors) avait inauguré la C.I.D., "composition interurbaine directe », et publié un dépliant bilingue intitulé En prévision de la C.I.D. Ce sigle était la forme française de D.D.D., Direct Distance Dialing. On remarquera que la traduction s'était faite à partir de to dial, rendu par composer. Plusieurs personnes, dont l'auteur de ces lignes, firent alors observer que ce n'était pas ainsi qu'il aurait fallu procéder et que de toute façon la compagnie aurait intérêt à s'enquérir de la désignation utilisée en France pour cette nouvelle 
invention. Il fallut un certain temps pour vaincre la résistance des auteurs de C.I.D. et pour obtenir l'adoption de automatique interurbain. Soit dit en passant, on trouve dans les textes français «interurbain automatique» aussi bien que « automatique interurbain ». La différence est négligeable. Toutefois on peut dire que, historiquement, l'automatique, fonctionnant d'abord à l'intérieur des localités, a été étendu aux communications interurbaines, ce qui peut justifier une certaine préférence pour automatique interurbain.

On entend de moins en moins, dans les bureaux tout au moins, « renverser les charges ». L'implantation de la formule à frais virés est facilitée par la question rituelle de la téléphoniste «Acceptez-vous les frais?»

On remarque aussi un progrès dans l'emploi de poste pour «local» ou « extension». De plus il semble bien qu'un nouvel usage soit en train de s'instituer dans l'épellation des numéros, les quatre derniers chiffres constituant alors deux nombres de deux chiffres : 31.43 au lieu de 3-1-4-3.

L'administration des postes imprime PAR EXPRÈS et non « livraison spéciale » et l'on note chez les usagers québécois une certaine désaffection à l'égard de «P.Q. » et de «Qué. » au profit de Québec en toutes lettres.

Dans la notation des heures, on constate l'usage grandissant depuis une dizaine d'années, de la notation de 0 à 24 , ce qui a pour effet de débarrasser la graphie des deux 0 , de «hres » et de P.M. Sur la plupart des convocations ou invitations que l'on reçoit, on lit, par exemple, 17 heures, 18 heures, 20 heures, etc. Dans ce domaine, les Forces armées (sous une forme un peu différente), Air Canada et les compagnies de chemins de fer ont donné le bon exemple et fait beaucoup pour habituer le public à cette notation depuis longtemps en usage sur le continent européen. Cependant on s'aperçoit que le passage d'une notation à l'autre n'est pas aussi facile qu'il y paraît. Certains s'embrouillent et combinent des éléments de chacune: « 18.30 heures ", alors qu'il est si simple d'écrire $18 \mathrm{~h} 30$. En un cas on a même relevé « 19 heures et demie», le scripteur ne s'étant pas avisé qu'entre 0 et 24 il n'y a plus ni quart ni demie.

Québec voulant affirmer son identité politique et culturelle, il était inévitable que son vocabulaire politique s'en trouvât modifié. Il est devenu celui d'un État plutôt que d'une Province, comme l'indique d'une façon très significative le déclin des mots province et provincial. Dans ce qui suit, on ne considérera, bien entendu, que les incidences langagières de changements dont nous n'avons pas à juger le bien-fondé. Le Musée provincial est devenu le Musée du Québec, et la police provinciale la Sûreté du Québec. Très caractéristique à cet égard est le changement de désignation des Archives de la Province et du titre des rapports annuels qui leur sont consacrés. De 1920 à 1960 quarante volumes réunissent les rapports de l'Archiviste de la province de Québec. Le 41 $1^{\mathrm{e}}$ volume, en 1963, porte le titre de Rapport des Archives du Québec. Depuis, le Bureau des archives s'est transformé en Archives nationales, avec à leur tête un conservateur et non un archiviste. Sur certains points, ce glissement de «la Province» au «Québec»se prolonge, comme on vient de le voir, jusqu'à l'emploi de national. Ce dernier adjectif surprend l'étranger francophone qui associe national à un État indépendant et trouve curieux qu'il y ait une Bibliothèque nationale et une Assemblée 
nationale dans une entité politique qui n'est pas souveraine. À cela on peut répondre que nation et nationalité n'ont pas tout à fait la même valeur au Québec qu'en France.

Depuis 1951 le Trésorier de la Province s'appelle le ministre des Finances, et en 1965 le Procureur général a pris le titre de ministre de la Justice. Il y a encore un Orateur à Ottawa, mais à Québec les débats de l'Assemblée sont dirigés par un Président. La Commission du Service civil est devenue celle de la Fonction publique; un "bill» s'appelle officiellement un projet de loi et l'Imprimeur de la Reine est devenu l'Éditeur officiel du Québec. Une désignation aussi ancrée dans l'usage que celle de «Commission des liqueurs》 a fait place, officiellement du moins, à Régie, puis à Société des alcools. Au ministère des Transports «l'enregistrement des véhicules moteurs » s'appelle maintenant l'immatriculation des véhicules automobiles. On note aussi, dans nombre de municipalités, la substitution de ville à «cité ».

Ces changements d'ordre administratif s'accompagnent nécessairement d'un renouvellement du vocabulaire, qui va dans le sens de la refrancisation. Certains mots sont écartés, d'autres s'installent, qui ne s'employaient pas auparavant. Les positions très fortes encore aujourd'hui de l'anglicisme «corporation » sont entamées par la diffusion naissante de société dans des appellations telles que Société Radio-Canada et Société des alcools. C'est une modeste tête de pont pour un terme auquel les légistes tendent à faire grise mine, mais qui est susceptible de s'élargir.

Depuis la révolution tranquille et nonobstant l'ambiguïté qui peut en résulter, l'adjectif québécois s'applique au pays et non pas seulement à sa capitale. En fait on se rend compte en lisant des textes antérieurs à 1960 que cette évolution de l'adjectif québécois et de son substantif était déjà amorcée au cours de la décennie qui a précédé la guerre. Et nous avons maintenant franco-québécois, le québécois (au sens de parler), québéciser (par exemple «québéciser les manuels français ») et même «québécitude ». M. Jean-Jacques Servan-Schreiber s'est fait vertement reprendre en 1971 par les étudiants de l'université Laval quand, prenant la parole devant eux, il les a appelés « Canadiens français».

\section{Conclusion}

Les exemples qui précèdent, et qui sont loin d'être exhaustifs, tendent à montrer qu'il y a actuellement une modernisation de la terminologie courante et que cette modernisation tend à diminuer l'écart (dont il ne faut d'ailleurs pas exagérer l'importance) entre le franco-québécois et le français international.

Parce que ces exemples reposent sur l'obseryation individuelle, ils ne permettent pas de mesurer l'étendu de la refrancisátion. Il est néanmoins utile de noter l'émergence de certains vocables, même si leur emploi reste encore sporadique. Et, comme on doit s'y attendre, la généralisation d'un mot dans des textes imprimés ne signifie pas qu'il est entré dans l'usage parlé. Bien qu'en France le système métrique soit vieux de près de deux cents ans, les mots livre et sou n'ont pas encore été chassés de l'usage oral par demi-kilo et centime. De semblable 
façon on entendra sans doute encore longtemps des Québécois dire qu'ils vont à la Commission des liqueurs ou qu'ils doivent s'occuper de renouveler leur enregistrement (de voiture). Les habitudes langagières, comme les autres, ne changent que graduellement.

Il faut d'ailleurs garder présents à l'esprit les obstacles à surmonter pour que la refrancisation s'épanouisse vraiment. Trois catégories de personnes n'y aident guère, même si elles ne la contrarient pas ouvertement. Les légistes répugnent à retoucher la rédaction d'un texte de loi; les hommes d'affaires hésitent à appeler les choses autrement, si cela risque d'éloigner la clientèle; enfin, les scientifiques misent surtout sur l'anglais pour la diffusion de leurs travaux. Dans ce dernier cas, il s'agit plutôt de l'extension du français que de son amélioration.

En outre, d'une façon générale, l'évolution du français québécois est ralentie par la crainte de se singulariser ou de n'être pas compris. En fait, l'expérience montre que le public accepte plus facilement les changements qu'on ne le croirait, ce qui ne veut pas dire qu'il les adopte pour son usage personnel. Sur ce point, il faut tenir compte de l'insécurité linguistique qui est de règle dans les pays bilingues et du flottement qu'elle provoque dans le sentiment que les usagers ont de leur langue ; on a l'impression qu'ils ont parfois peur des mots.

Il est malaisé d'évaluer l'importance relative des facteurs de refrancisation. On notera d'abord l'influence des «media », beaucoup plus celle de la radio et de la télévision que celle de la presse. Elle se sent surtout dans le français parlé, dans le soin apporté à l'élocution dans les émissions pour satisfaire aux exigences du métier d'annonceur. Ensuite et en bonne place intervient le développement du secteur technique (dont le barrage de Manic est sans doute l'une des réalisations les plus spectaculaires) et les besoins terminologiques en français qui en sont la conséquence. Et, bien entendu, les traducteurs ont joué un rôle important. Depuis 1940 des associations de traducteurs sont nées au Québec et dans l'Ontario, et la préparation à la profession a été rendue méthodique par la création d'un enseignement donné d'abord uniquement par cours du soir, auxquels se sont ajoutés, depuis quelques années, des programmes d'études universitaires spécialisés en traduction. La revue Meta, qui permet aux traducteurs de se documenter et de prendre conscience de leur importance, a déjà derrière elle vingt années bien remplies. Sans aller jusqu'à prétendre que tous les traducteurs actuellement en exercice ont été touchés par l'esprit nouveau, on peut du moins affirmer que la profession a été fortement sensibilisée aux équivalences de traduction et à la recherche du mot juste. Les vocabulaires spécialisés de l'Office de la langue française se révèlent dans ce domaine particulièrement efficaces. Si on tient aussi compte de certaines réalisations dans le domaine de l'informatique, on peut dire que le Canada est à la pointe de la recherche en terminologie dans l'ensemble de la francophonie. De plus, cette recherche ne se fait pas en vase clos; elle est stimulée par les contacts avec les autres pays francophones. À cet égard, la fin de l'isolement constitue le facteur qui sous-tend tous les autres.

L'effet de tous ces facteurs sur les noms de choses, de processus, d'institutions, en un mot sur l'élément terminologique du langage, est incontestable. Il est moins positif dans le domaine de la phraséologie et des tournures de phrase. 
Cependant même là des améliorations se font jour. Dans un récent dépliant bilingue on trouve côte à côte you may be eligible for et « vous pourriez avoir droit à ». Il fut un temps où le traducteur aurait gardé «éligible ». Sans doute, une hirondelle ne fait pas le printemps, mais, justement, il n'y a pas qu'une hirondelle.

Toutefois l'élargissement et la consolidation de la tendance que nous venons de décrire restent subordonnés à des facteurs d'ordre psychologique. On peut les résumer en un mot qui est motivation. Au passif de la situation, il convient, en toute objectivité, de faire figurer une certaine indifférence à la qualité du français écrit ou parlé. À la peur de ne pas se faire comprendre (citée plus haut) s'ajoute le sentiment qu'il suffit de se faire comprendre. Dans cette perspective la précision et la correction risquent d'apparaitre comme des ornements dont on peut se passer. Et à cet égard, le souci actuel de beaucoup d'enseignants de mettre l'accent sur la créativité et sur l'élocution orale (qui en fait prime la facilité plutôt que la correction) nuit à la rigueur qui, seule et dans quelque pays que ce soit, peut assurer, là où il ne vient pas naturellement, le maniement correct et idiomatique de la langue nationale. Il ne suffit pas de parler spontanément sa langue maternelle, il faut l'apprendre. Le français des Québécois sera ce qu'ils voudront qu'il soit.

Jean Darbelnet 